\title{
Strong electronic correlation in the hydrogen chain: A variational Monte Carlo study
}

\author{
Lorenzo Stella, ${ }^{1,2, *}$ Claudio Attaccalite, ${ }^{3}$ Sandro Sorella, ${ }^{4}$ and Angel Rubio ${ }^{1,5}$ \\ ${ }^{1}$ Nano-Bio Spectroscopy group and ETSF Scientific Development Centre, Departamento Física de Materiales, Universidad del País Vasco, \\ Centro de Física de Materiales CSIC-UPV/EHU-MPC and DIPC, Avenida Tolosa 72, E-20018 San Sebastián, Spain \\ ${ }^{2}$ Department of Physics and Astronomy and London Centre for Nanotechnology, University College London, Gower Street, \\ London WC1E 6BT, United Kingdom \\ ${ }^{3}$ Institut Néel, CNRS/UJF 25 rue des Martyrs BP 166, Bâtiment D, F-38042 Grenoble cedex 9, France \\ ${ }^{4}$ SISSA, Via Bonomea 265, I-34136 Trieste, Italy and Dipartimento di Fisica, Università di Trieste, strada Costiera 11, I-34151 Trieste, Italy \\ ${ }^{5}$ Fritz-Haber-Institut der Max-Planck-Gesellschaft, Berlin, Germany
}

(Received 29 July 2011; published 14 December 2011)

\begin{abstract}
In this paper, we report a fully ab initio variational Monte Carlo study of the linear and periodic chain of hydrogen atoms, a prototype system providing the simplest example of strong electronic correlation in low dimensions. In particular, we prove that numerical accuracy comparable to that of benchmark densitymatrix renormalization-group calculations can be achieved by using a highly correlated Jastrow-antisymmetrized geminal power variational wave function. Furthermore, by using the so-called "modern theory of polarization" and by studying the spin-spin and dimer-dimer correlations functions, we have characterized in detail the crossover between the weakly and strongly correlated regimes of this atomic chain. Our results show that variational Monte Carlo provides an accurate and flexible alternative to highly correlated methods of quantum chemistry which, at variance with these methods, can be also applied to a strongly correlated solid in low dimensions close to a crossover or a phase transition.
\end{abstract}

DOI: 10.1103/PhysRevB.84.245117

PACS number(s): 71.30.+h, 73.21.Hb, 31.15.A-, 02.70.Ss

\section{INTRODUCTION}

The homogeneous (i.e., equispaced), linear, and periodic chain of hydrogen atoms (hereafter, the $\mathrm{H}$ chain) is commonly believed to be the simplest physical system described by the one-band, periodic, one-dimensional (1D) Hubbard Hamiltonian $^{1}$ [see Eq. (11)]. This Hamiltonian is exactly solvable $^{2}$ and its solution predicts the $\mathrm{H}$ chain to be always a Mott-Hubbard (i.e., a strongly correlated) insulator. Indeed, it seems reasonable to model the $\mathrm{H}$ chain by including only the $1 s$ orbitals and by neglecting the long-range tail of the Coulomb interaction, especially for large interatomic distances. As a consequence, the $\mathrm{H}$ chain has been intensively studied to benchmark $a b$ initio approaches to strong electronic correlation $^{3-6}$ despite the fact that this atomic chain is not directly observable due to the well-known Peierls' instability.

Previous $a b$ initio studies-mostly using methods of quantum chemistry - have been focused on finite (i.e., not periodic) linear chains, only. However, periodic boundary conditions (PBCs) analogous to the Born-von Kármán boundary conditions used in solid-state physics are better suited to investigate phase transitions or crossovers. Indeed, unwanted edge effects are avoided by using PBCs and a speedup of the convergence to the thermodynamic limit, i.e., the limit of infinite linear chains, is expected. ${ }^{7,8}$ Hence an $a b$ initio description of the low-energy physics of a properly periodic $\mathrm{H}$ chain is still missing. In this paper we provide an exhaustive, fully ab initio ${ }^{9}$ variational description of periodic chains by using the same kind of variational wave function for both the weakly and the strongly correlated regimes, i.e., for both small and large interatomic distances.

From previous studies, it is known that in the strongly correlated regime-i.e., for interatomic distances $a$ larger than a certain critical distance $a_{c}$-the ground state of the finite $\mathrm{H}$ chains is characterized by a huge degeneracy of the natural orbitals, ${ }^{3,4}$ which leads to an almost uniform natural orbital population, narrowly dispersed around $1 .^{6}$ This behavior has to be contrasted with the weakly correlated regime $\left(a<a_{c}\right)$ for which already the restricted Hartree-Fock reference- that yields either a doubly occupied or empty natural orbital-is quite accurate. ${ }^{10}$

In many cases ${ }^{4}$ part of the static correlation that characterizes the strongly correlated regime can be effectively recovered by means of an unrestricted Hartree-Fock calculation, or equivalently, by means of a spin-polarized density-functional theory calculation within the local-density approximation. ${ }^{1}$ Although justified for finite systems, a spin-polarized approach implies a mean-field antiferromagnetic order, which cannot be trusted in the thermodynamic limit, because true antiferromagnetism is not possible for $1 \mathrm{D}$ solids. ${ }^{11}$

The density-matrix renormalization group (DMRG) provides an, in principle exact, algorithm to compute the electronic structure of $1 \mathrm{D}$ and almost-1D systems, although in practice limited by the size of the orbital basis set. ${ }^{12}$ DMRG works very efficiently also when other highly correlated methods fail, e.g., configuration interaction ${ }^{13}$ is not applicable if the system size is too large and the standard coupled cluster singles and doubles plus perturbative triples becomes unstable in one dimension for large interatomic distances. ${ }^{3}$ Nevertheless, even the very favorable numerical efficiency of DMRG is lost for a gapless (i.e., metallic) chain. In this case, also the Moller-Plesset perturbative approach is not straightforwardly applicable due to the numerical issues triggered by the vanishing small gap.

\section{COMPUTATIONAL METHODS}

Among the possible alternatives to standard quantum chemical approaches, ${ }^{4,6}$ one can consider nonperturbative 
quantum Monte Carlo (QMC) methods like variational Monte Carlo (VMC) or the, in principle more accurate, diffusion Monte Carlo (DMC). ${ }^{14}$ In fact, a direct application of DMC to a homogeneous chain raises some ergodicity issues when the electrons are very localized about the nuclei, i.e., in the strongly correlated regime. ${ }^{15}$ As a consequence, to date only alternating (i.e., dimerized) chains have been investigated by means of DMC. ${ }^{16}$ On the other hand, VMC can be made ergodic by tailoring the sampling and by improving the variational many-body wave function, so that it remains very effective also close to crossovers and phase transitions. ${ }^{7,8}$

There have been dramatic theoretical advances in the quality of the variational wave function during the last decade, so that it is now possible to achieve chemical accuracy for atoms, ions, small molecules, and even periodic systems. ${ }^{17-21}$ These quantitative improvements have been made possible by new stochastic optimization techniques, which can optimize variational wave functions depending on hundreds of free parameters. $^{22}$

In our variational calculations, we have used a Jastrowantisymmetrized geminal power (JAGP) variational wave function for $N$ (even) interacting electrons, ${ }^{18,19}$

$$
\Psi_{N}(\vec{R})=J(\vec{R}) \mathcal{A} \prod_{i=1}^{N / 2} \Phi\left(\vec{r}_{i}^{\uparrow}, \vec{r}_{i}^{\downarrow}\right),
$$

where $\vec{R}=\left\{\vec{r}_{1}^{\uparrow}, \ldots, \vec{r}_{N / 2}^{\uparrow}, r_{1}^{\downarrow}, \ldots, \vec{r}_{N / 2}^{\downarrow}\right\}$ is the $3 N$-dimensional coordinate vector, $\mathcal{A}$ is the antisymmetrization operator, $\Phi\left(\vec{x}^{\uparrow}, \vec{y}^{\downarrow}\right)$ is a symmetric function describing a singlet electron pair, and

$$
J(\vec{R})=\exp \sum_{i, j}^{N}\left[u\left(r_{i, j}\right)+f\left(\vec{r}_{i}, \vec{r}_{j}\right)\right]
$$

is the Jastrow factor.

By using Eq. (1), one can accurately describe both static and dynamics correlations. Indeed, the antisymmetrized geminal power, $\mathcal{A} \prod_{i=1}^{N / 2} \Phi\left(\vec{r}_{i}^{\uparrow}, \vec{r}_{i}^{\downarrow}\right)$, provides a very compact multideterminant reference, while the Jastrow factor takes into account the dynamic correlation by means of the following: (i) A short-range homogeneous electron-electron interaction through the term $u\left(r_{i, j}\right)$, which just depends on the distance, $r_{i, j}=\left|\vec{r}_{i}-\vec{r}_{j}\right|$, between paired electrons. ${ }^{14}$ (ii) The inhomogeneous electron-electron-nucleus and electron-electronnucleus-nucleus interactions through the term $f\left(\vec{r}_{i}, \vec{r}_{j}\right)$, which depends separately on the coordinates of the paired electrons, and it can also describe long-range electronic correlations.

Results shown in this paper have been obtained using a short-range homogeneous Jastrow factor, $u\left(r_{i, j}\right)=(1 / 2 b)(1-$ $\left.e^{-b r_{i, j}}\right)$. In addition, to assess the sensitivity of these results on the details of the wave function, we have also considered a long-range homogeneous Jastrow factor, $u\left(r_{i, j}\right)=r_{i, j} / 2(1+$ $b r_{i, j}$ ) (see Fig. 4). In both cases, $b$ is a variational parameter, and the electronic cusp conditions ${ }^{14}$ are automatically satisfied.

The functions $\Phi\left(\vec{x}^{\uparrow}, \vec{y}^{\downarrow}\right)$ in Eq. (1) and $f(\vec{x}, \vec{y})$ in Eq. (2) are expanded using (in principle different) nonorthogonal atomic orbitals, ${ }^{23}\left\{\phi_{i}\right\}$ and $\left\{\varphi_{i}\right\}$ so that

$$
\begin{aligned}
\Phi\left(\vec{x}^{\uparrow}, \vec{y}^{\downarrow}\right) & =\sum_{\alpha, \beta} \lambda_{\alpha, \beta} \varphi_{\alpha}\left(\vec{x}^{\uparrow}\right) \varphi_{\beta}\left(\vec{y}^{\downarrow}\right), \\
f(\vec{x}, \vec{y}) & =\sum_{\alpha, \beta} g_{\alpha, \beta} \phi_{\alpha}(\vec{x}) \phi_{\beta}(\vec{y}) .
\end{aligned}
$$

In particular, up to $3 s$ orbitals for the geminal part and $2 s 2 p$ for the Jastrow part have been considered in this work. ${ }^{24}$

In principle, all the entries of the matrices $\lambda_{\alpha, \beta}$ and $g_{\alpha, \beta}$ in Eq. (3) are variational parameters to be optimized. However, by taking advantage of the symmetries of the periodic linear chain, ${ }^{21}$ and by using an alternative, minimal expansion in terms of molecular orbitals, ${ }^{19}$ the actual number of independent variational parameters to optimize is reduced, so that the optimization can be effectively performed by the method described in Ref. 22.

All the VMC calculations have been performed by using the TurboRVB package, ${ }^{25}$ starting from a density-functional theory (in local-density approximation) calculation employing the same orbital basis set $\left\{\varphi_{i}\right\}$ of the geminal part [see Eq. (3)]. This preliminary step is done to speed up the convergence of the following VMC optimization, while avoiding an uncontrolled bias.

Finally, we have used a supercell with $\mathrm{PBCs}^{14}$ in all three Cartesian directions to model the periodic linear chain. To avoid unphysical self-interaction of the chain with its periodic replicas, the transverse dimensions of the supercell have been taken as $\min (16 a, 80)$ (a.u.), where $a$ is the interatomic distance. According to the supercell formalism, the thermodynamic limit, $N \rightarrow \infty$, can be achieved by increasing the number $N$ of $\mathrm{H}$ atoms in the supercell. In particular, where not otherwise indicated, by $\mathrm{H}_{N}$ we mean a periodic chain with $N$ atoms in the supercell.

To identify the weakly and the strongly correlated regimes, we have used the so-called "modern theory of polarization. ${ }^{26}$ This theory also provides a way to discriminate between a metal and an insulator alternative to the knowledge of the (many-body) charge gap, which in fact is not accessible by a variational ground-state calculation.

In practice, by $\mathrm{VMC}$ one computes the expectation values of the complex polarization, $z_{N},{ }^{16}$

$$
z_{N}=\left\langle\Psi_{N}\left|e^{i(2 \pi / L) \sum_{i} r_{i}^{\|}}\right| \Psi_{N}\right\rangle
$$

where $r_{i}^{\|}$is the component of $\vec{r}_{i}$ parallel to the chain axis. Then the electronic localization length $\lambda_{N}$ is obtained as

$$
\lambda_{N}=\left(\frac{L}{2 \pi}\right) \sqrt{-\frac{\ln \left|z_{N}\right|^{2}}{N}},
$$

where $L$ is the longitudinal dimension of the supercell and $N$ is the number of $\mathrm{H}$ atoms in the supercell.

From previous studies, ${ }^{3,4,6}$ one expects a huge degeneracy of the natural orbitals when the electrons are very localized about the nuclei, i.e., when $\lambda_{N} / a \ll 1$. Besides, the theory says that, in the thermodynamic limit, $N \rightarrow \infty$, a metal is characterized by a vanishing modulus of the complex polarization, $\left|z_{N}\right| \rightarrow 0$, while in the insulating case $\left|z_{N}\right| \rightarrow 1^{7,8}$ 


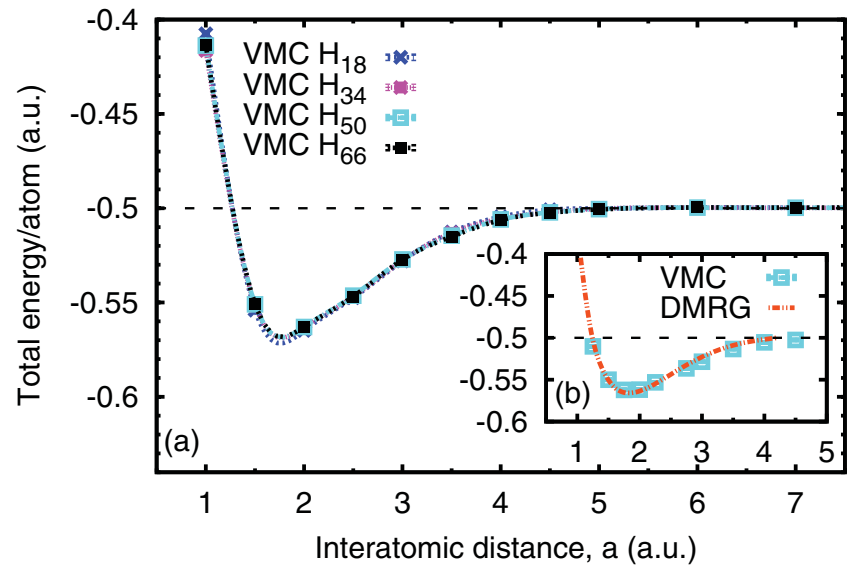

FIG. 1. (Color online) (a) Total energy per atom as a function of the interatomic distance from VMC calculations of periodic chains with 18, 34, 50, and $66 \mathrm{H}$ atoms in the supercell. (Data are almost superimposed at the scale of this figure; see also Table I.) (b) Comparison between the total energy per atom of a finite $\mathrm{H}_{50}$ chain obtained by VMC and DMRG (Ref. 3).

\section{RESULTS}

In Fig. 1(a), we show the convergence of the total energy per atom by increasing the number of $\mathrm{H}$ atoms per supercell for several interatomic distances. We note that the $\mathrm{H}_{50}$ periodic $\mathrm{H}$ chain is already well converged at the scale of this figure. To follow the fine detail of the convergence, the values of the total energy per atom details have been also listed in Table I.

In Fig. 1(b), a direct comparison between the VMC total energy for the $\mathrm{H}_{50}$ finite chain and the benchmark DMRG results obtained by using a STO- $6 G$ basis $\operatorname{set}^{3}$ demonstrates the accuracy of our optimized JAGP variational wave function. ${ }^{27}$ In this case, to have a fair comparison against the DMRG data, PBCs have not been employed to obtain the VMC results showed in Fig. 1(b). The difference between the total energy of $\mathrm{H}_{50}$ chains with and without PBCs and the same interatomic distance is of the order of few mHa per atom.

Having verified the quality of the variational wave function, in Fig. 2(a) we plot the electronic localization length $\lambda_{N}$ in units of the interatomic distance $a$ as a function of $a$. For all

TABLE I. Total energy per atom as a function of the interatomic distance $a$ for the same periodic chains of Fig. 1(a). The VMC error on the last digit is indicated in parentheses.

\begin{tabular}{ccccc}
\hline \hline$a$ & $\mathrm{H}_{18}$ & $\mathrm{H}_{34}$ & $\mathrm{H}_{50}$ & $\mathrm{H}_{66}$ \\
\hline 1.0 & $-0.40751(4)$ & $-0.41639(3)$ & $-0.41380(3)$ & $-0.41358(2)$ \\
1.5 & $-0.55402(2)$ & $-0.55156(1)$ & $-0.55099(1)$ & $-0.55070(1)$ \\
2.0 & $-0.56480(2)$ & $-0.56329(1)$ & $-0.56296(1)$ & $-0.56284(1)$ \\
2.5 & $-0.54747(2)$ & $-0.54699(1)$ & $-0.54639(1)$ & $-0.54682(1)$ \\
3.0 & $-0.52796(2)$ & $-0.52770(2)$ & $-0.52717(1)$ & $-0.52727(1)$ \\
3.5 & $-0.51263(3)$ & $-0.51308(2)$ & $-0.51459(2)$ & $-0.51508(1)$ \\
4.0 & $-0.50458(3)$ & $-0.50556(4)$ & $-0.50599(2)$ & $-0.50626(1)$ \\
4.5 & $-0.50080(3)$ & $-0.50206(1)$ & $-0.50222(1)$ & $-0.50237(1)$ \\
5.0 & $-0.50014(2)$ & $-0.50029(1)$ & $-0.50047(1)$ & $-0.50063(1)$ \\
6.0 & $-0.49962(1)$ & $-0.49971(1)$ & $-0.49972(1)$ & $-0.49965(1)$ \\
7.0 & $-0.49980(1)$ & $-0.49981(1)$ & $-0.49979(1)$ & $-0.49972(1)$ \\
\hline \hline
\end{tabular}

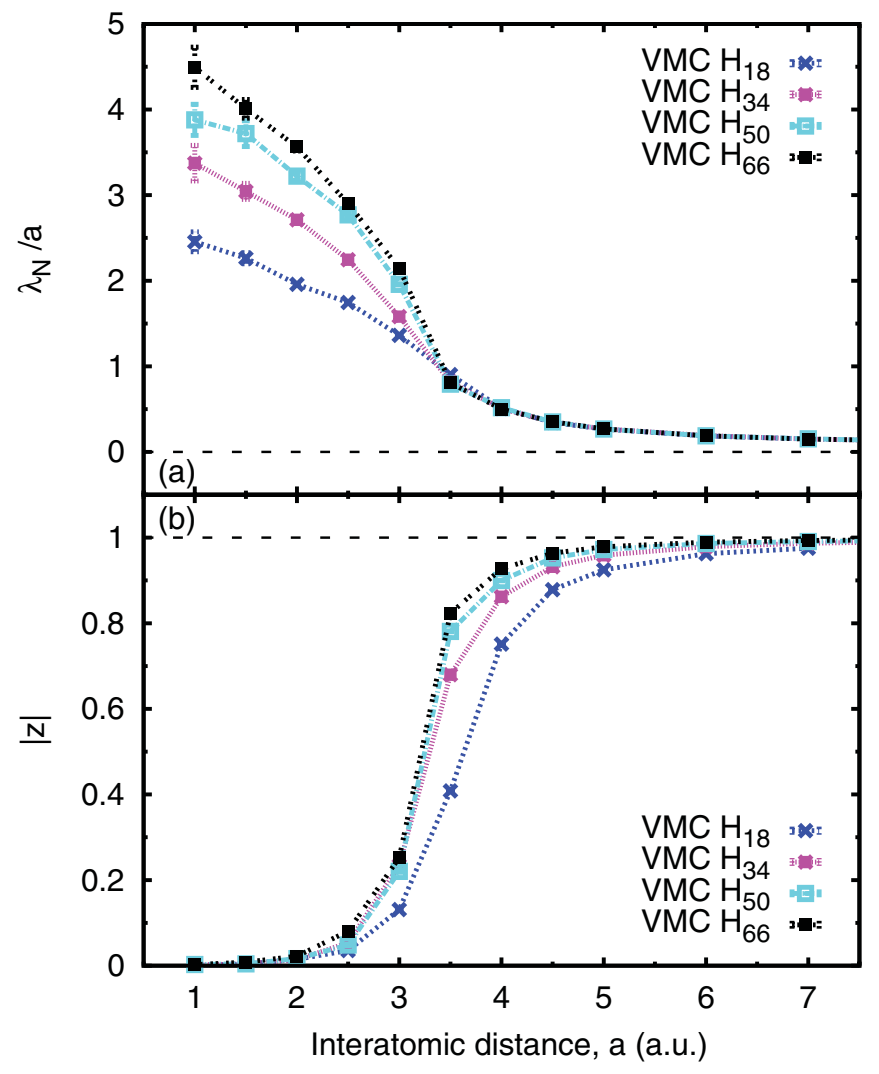

FIG. 2. (Color online) (a) Electronic localization length $\lambda_{N}$ divided by the interatomic distance $a$ as a function of $a$, for the same chains of Fig. 1(a). (d) Modulus of the complex polarization $\left|z_{N}\right|$ as a function of the interatomic distance for the same chains of (c).

the supercells considered, we find that

$$
\lambda_{N} / a \propto\left\{\begin{array}{ccc}
\left|a-a_{c}\right|^{\eta} & \text { if } & a<a_{c}, \\
a^{-1} & \text { if } & a>a_{c},
\end{array}\right.
$$

where $\eta \simeq 0.5$ and $a_{c} \simeq 3.5$ (a.u.). This critical behavior is also in agreement with the sudden switch from $|z| \simeq 0$ to $|z| \simeq 1$ visible in Fig. 2(b), i.e., to the crossover between a (finite-size) metal and an insulator, namely a Mott-Hubbard insulator. ${ }^{1}$

To further characterize the nature of the weakly and strongly correlated regimes of the $\mathrm{H}$ chain, we have investigated the spin-spin,

$$
f_{s s}(i-j)=\left\langle\Psi_{N}\left|\hat{S}_{z}^{(i)} \hat{S}_{z}^{(j)}\right| \Psi_{N}\right\rangle,
$$

and the dimer-dimer,

$$
f_{d d}(i-j)=\left\langle\Psi_{N}\left|\hat{S}_{z}^{(i)} \hat{S}_{z}^{(i+1)} \hat{S}_{z}^{(j)} \hat{S}_{z}^{(j+1)}\right| \Psi_{N}\right\rangle
$$

correlation functions, where $\hat{S}_{z}^{(i)}$ measures the transverse component of the electronic spin about the $i$ th $\mathrm{H}$ atom of the chain. By neglecting logarithmic corrections, we have fitted these functions by ${ }^{28}$

$$
\begin{gathered}
f_{s s}(i-j)=\frac{a_{s s}}{(i-j)^{2}}+b_{s s} \frac{\cos [\pi(i-j)]}{(i-j)^{K_{s s}}}, \\
f_{d d}(i-j)=a_{d d}+b_{d d} \frac{\cos [\pi(i-j)]}{(i-j)^{K_{d d}}}
\end{gathered}
$$




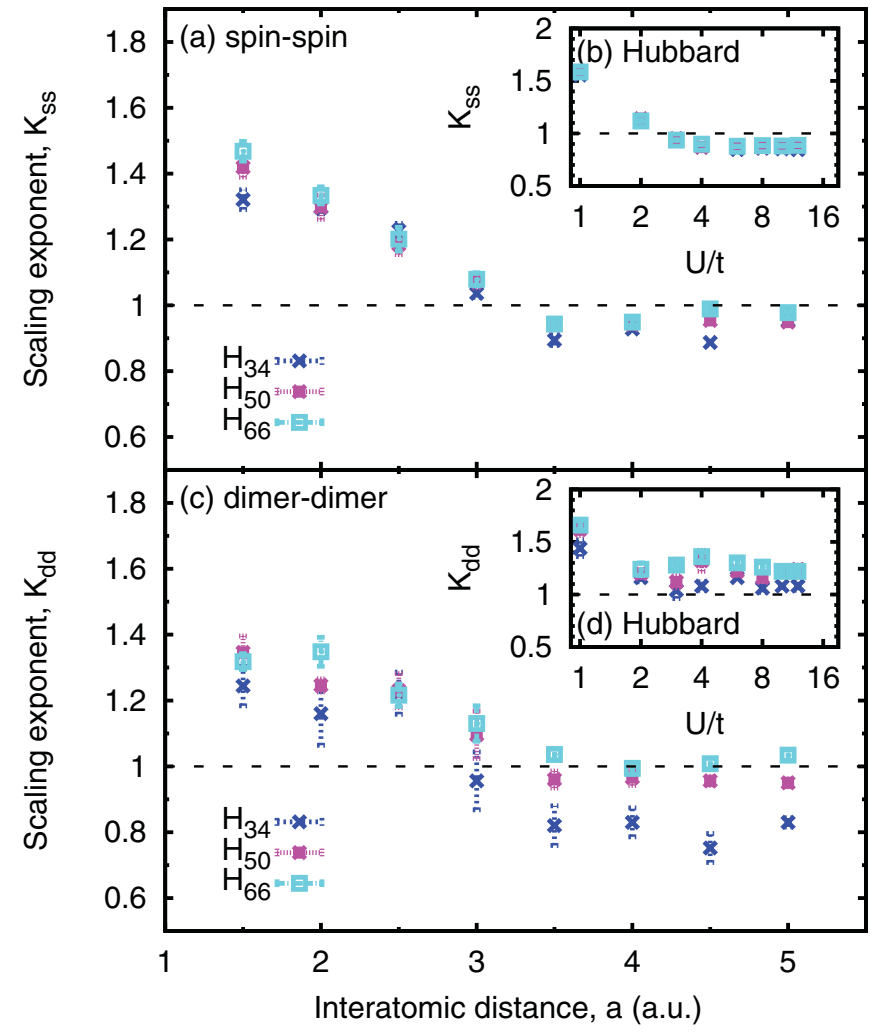

FIG. 3. (Color online) (a) Scaling exponent $K_{s s}$ of the spin-spin correlation function [see Eq. (9)] as a function of the interatomic distance, for same $\mathrm{H}$ chains of Figs. 1 and 2. (b) Scaling exponent $K_{s s}$ of the Hubbard model with a corresponding number of sites (see text). (Data are superimposed at the scale of this figure.) (c),(d) Same as (a) and (b), but for the dimer-dimer correlation function [see Eq. (10)]

for the spin-spin and the dimer-dimer cases, respectively. The parameters, $a_{s s}, b_{s s}, K_{s s}, a_{d d}, b_{d d}$, and $K_{d d}$ have been fitted independently for each value of the interatomic distance $a$ and the number of $\mathrm{H}$ atoms in the supercell.

Results for the scaling exponents, $K_{s s}$ and $K_{d d}$, of the $\mathrm{H}$ chain are reported in Figs. 3(a) and 3(c), respectively. For comparison, in Figs. 3(b) and 3(d) we show the scaling exponents obtained by solving numerically ${ }^{29}$ the $N$-site Hubbard model Hamiltonian ${ }^{1}$ with PBCs (i.e., the simulation cell is folded so that the $(j+N)$ th and $j$ th sites represent the same atom),

$$
H=-t \sum_{j=1}^{N} \sum_{\sigma=\uparrow, \downarrow}\left(c_{j, \sigma}^{\dagger} c_{j+1, \sigma}+c_{j+1, \sigma}^{\dagger} c_{j, \sigma}\right)+U \sum_{j=1}^{N} n_{j, \uparrow} n_{j, \downarrow},
$$

with a number of sites $N$ correspondent to the number of $\mathrm{H}$ atoms in the chain supercell. In Eq. (11), the creation (annihilation) operator $c_{j, \sigma}^{\dagger}\left(c_{j, \sigma}\right)$ creates (annihilates) an electron of spin $\sigma$ at site $j$, while $n_{j, \sigma}=c_{j, \sigma}^{\dagger} c_{j, \sigma}$. Since one expects (for $U$ fixed) that $\ln (U / t) \propto a$, we have shown the Hubbard exponents as a function of $U / t$ using a semilog plot.

The behavior of the scaling exponent $K_{s s}$ is very similar in the two cases, i.e., the $\mathrm{H}$ chain in Fig. 3(a) and the Hubbard model in Fig. 3(b). However, small but noticeable discrepancies between the $\mathrm{H}$ chain and the Hubbard model for

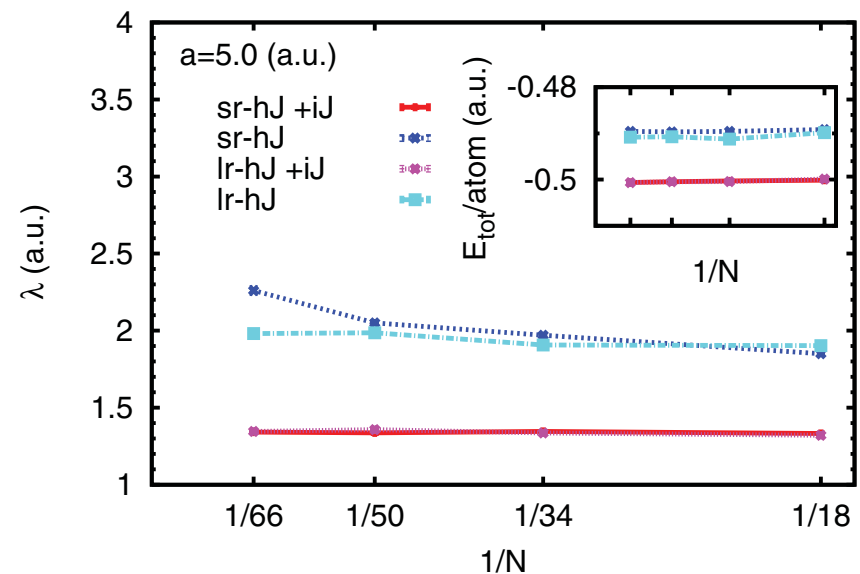

FIG. 4. (Color online) (a) Localization length as a function of the inverse of the number of $\mathrm{H}$ atoms in the supercell for different parametrizations of the JAGP variational wave function (see text). (Data "sr-hJ + iJ" and "lr-hJ + iJ" are superimposed at the scale of this figure; see also Table II.) (b) Corresponding total energy per atom.

the scaling exponent $K_{d d}$ are found by comparing Figs. 3(c) and 3(d).

For large interatomic distance $a$, both the H-chain exponents behave as expected for the Hubbard model (neglecting logarithmic corrections), i.e., $K_{d d} \sim K_{s s} \sim 1{ }^{28}$ Less conclusive results can be inferred from the weakly correlated regime for $a<a_{c}$. Finite-size effects are responsible for the deviation of the scaling exponents from their thermodynamic values in the case of the Hubbard model. The same effects also mask possible discrepancies in the thermodynamic limit of the $\mathrm{H}$ chain and the Hubbard model. Further numerical investigations are needed to provide conclusive results on a possible metal-insulator transition at a finite value $a_{c}$ of the interatomic distance of the $\mathrm{H}$ chain (see Sec. IV).

Finally, we investigate in more detail the capability of the variational JAGP wave function to describe the Mott-Hubbard insulating phase of the $\mathrm{H}$ chain for $a>a_{c}$. In particular, we focus on the $a=5.0$ (a.u.) case and we consider some variants of the JAGP variational wave function, Eq. (1).

In Fig. 4(a) we plot the electronic localization length $\lambda_{N}$ obtained by optimizing different JAGP variational wave functions. We consider the following cases (for the notation, see previous section): (i) sr-hJ + iJ, corresponding to Eq. (1) with short-range homogeneous Jastrow factor plus the inhomogeneous part. This is the standard case considered elsewhere in this paper; (ii) lr-hJ $+\mathrm{iJ}$, as the $s r-h \mathrm{~J}+\mathrm{iJ}$ wave function, but with a long-range homogeneous Jastrow factor, instead; (iii) sr-hJ, and (iv) lr-hJ, which are as the sr-hJ $+\mathrm{iJ}$ and $\mathrm{lr}-\mathrm{hJ}+\mathrm{iJ}$ wave functions, respectively, but without the inhomogeneous Jastrow factor, i.e., with $f\left(\vec{r}_{i}, \vec{r}_{j}\right)=0$.

We find that the localization length $\lambda_{N}$ is well converged at the scale of Fig. 4(a) if the inhomogeneous Jastrow factor is included, regardless of the choice of the homogeneous part. If the inhomogeneous Jastrow factor is not included, values of $\lambda_{N}$ almost twice as large are found and they slightly increase with the system size, showing that the homogeneous Jastrow alone is not enough to give an accurate description of the 
TABLE II. Total energy per atom as a function of the number, $N$, of $\mathrm{H}$ atoms in the supercell, for the same chains of Fig. 4(b). The $\mathrm{VMC}$ error on the last digit is indicated in parenthesis.

\begin{tabular}{ccccc}
\hline \hline$N$ & sr-hJ+iJ & lr-hJ+iJ & sr-hJ & lr-hJ \\
\hline 18 & $-0.50014(2)$ & $-0.49987(2)$ & $-0.48918(6)$ & $-0.48984(6)$ \\
34 & $-0.50029(1)$ & $-0.50042(1)$ & $-0.48956(4)$ & $-0.49123(3)$ \\
50 & $-0.50047(1)$ & $-0.50045(1)$ & $-0.48969(3)$ & $-0.49072(3)$ \\
66 & $-0.50063(1)$ & $-0.50066(1)$ & $-0.48956(3)$ & $-0.49082(3)$ \\
\hline \hline
\end{tabular}

Mott-Hubbard insulating phase. Values of the total energy per atom reported in Fig. 4(b) also confirm the relevance of the long-range, inhomogeneous Jastrow factor to improve the accuracy of the VMC description of a Mott-Hubbard insulator. To follow the fine detail of the convergence, the values of the total energy per atom have been also listed in Table II.

Our findings are in agreement with previous variational studies of lattice models with short-range interactions which showed that a correct description of the Mott-Hubbard insulating phase can be achieved only by combining a Gutzwiller projector and a long-range Jastrow factor. ${ }^{8,30}$

In fact, although the single $\mathrm{H}$ atoms are on average neutral, charge fluctuations that give, e.g., virtual $\mathrm{H}^{+}-\mathrm{H}^{-}$pairs are always possible. Such charge fluctuations are in fact strongly suppressed at large interatomic distance $a$ (and, in the Hubbard model, for large $U$ ). Therefore, at large $a$, in order to prevent an instability of the Mott-Hubbard insulator toward a metallic state driven by the quantum fluctuations, ${ }^{31}$ the $\mathrm{H}^{+}-\mathrm{H}^{-}$pairs must be bound. In the context of the Hubbard model, ${ }^{8,30}$ it has been demonstrated that such binding mechanism can be included in the variational wave function by means of an inhomogeneous Jastrow factor, analogous to the the second term in Eq. (2). Crucially, the matrix element $g_{\alpha, \beta}$ in the expansion of the inhomogeneous Jastrow factor [see Eq. (3)] can be nonvanishing also for pairs of orbitals (labeled by the Greek indices) that are far apart. Indeed, such long-range correlation is necessary to bind the virtual $\mathrm{H}^{+}-\mathrm{H}^{-}$pairs and, along with the homogeneous Jastrow factor, provides an accurate way to model electron localization in the MottHubbard insulating phase, as shown in Fig. 4(a).

\section{DISCUSSION AND CONCLUSIONS}

In this paper, we have investigated the ground state of a homogeneous, linear, and periodic chain of hydrogen atoms (or $\mathrm{H}$ chain) from first principles, by means of a state-of-the-art variational Monte Carlo approach. In fact, using a highly correlated Jastrow-antisymmetrized geminal power variational wave function allowed us to obtain a total energy per atom comparable to benchmark density-matrix renormalization-group calculations. ${ }^{3}$ Furthermore, by using the so-called "modern theory of polarization," 26 we have characterized the crossover between the weakly correlated (for small interatomic distances) and the strongly correlated regimes (for large interatomic distances) through a single, simple parameter, i.e., the electronic localization length. Our results extend to the properly periodic $\mathrm{H}$-chain previous results obtained by studying long, yet finite, chains. ${ }^{3,4,6}$ In particular, we confirmed that the crossover between the weakly and strongly correlated regimes of the $\mathrm{H}$ chain corresponds physically to a crossover between a (finite-size) metallic and an insulating phase. Finally, by studying the asymptotic behavior of the spin-spin and dimer-dimer correlation functions, we have also verified that the insulating phase of the real $\mathrm{H}$ chain is of the Mott-Hubbard type, as expected from the Hubbard model. ${ }^{1}$ Since the correct description of such a correlated insulator is beyond the possibility of density-functional theory in any of its conventional local or semilocal approximations, one can think of using our findings to devise an improved class of functionals. We are currently exploring this possibility.

Intriguingly, we have found small but noticeable deviations from the behavior predicted by the Hubbard model in the case of the scaling exponent of the dimer-dimer correlation function predicted by the Hubbard model. ${ }^{28}$ These deviations can be possibly due to the finite-size scaling or to a true discrepancy between the $\mathrm{H}$ chain and the 1D Hubbard model in the thermodynamic limit.

It will be interesting to check for possible new low-energy physics of the $\mathrm{H}$ chain at variance of the one-band, 1D Hubbard model predictions. These might be originated by the following: (i) the long-range Coulomb repulsion-indeed inefficiently screened in 1D systems-not included in the Hubbard model; (ii) the atomic orbital polarization-essential to describe noncovalent contribution to the bonding-not representable in terms of $1 s$ orbitals, only. In particular, relative simple elaborations of the Hubbard model, which just contain next-nearest-neighbor interaction, already predict a rich phase diagram even for a $1 \mathrm{D}$ system. ${ }^{8,32}$ Besides, it is known that long-range Coulomb repulsion can yield gapless charge excitations (plasmons) in one dimension, as observed in experiments. ${ }^{33}$

Of course, more accurate finite-size extrapolation is desirable, although not possible with our current computational resources. In particular, the use of diffusion Monte Carlo to improve the variational results might be also beneficial, but in practice still highly problematic due to the well-known ergodicity issues in dealing with strong electronic localization in $1 \mathrm{D}$ systems. ${ }^{15}$

In conclusion, given that the homogeneous, linear, and periodic chain of hydrogen atoms is becoming a standard test case for $a b$ initio approaches to strong electronic correlation, ${ }^{3,4,6}$ the results reported in this paper show that variational Monte Carlo (with a highly correlated Jastrow-antisymmetrized geminal power variational wave function) can provide an accurate and flexible alternative to highly correlated methods of quantum chemistry. Besides, and at variance with most of the methods of quantum chemistry, variational Monte Carlo can be successfully employed to study a strongly correlated solid in low dimensions close to a crossover or a phase transition. ${ }^{7,8}$

\section{ACKNOWLEDGMENTS}

This work has been performed under the HPC-EUROPA2 project (Project No. HPC08TH6KD) with the support of the European Commission - Capacities Area - Research Infrastructures and using HPC resources from GENCI-IDRIS (Project No. 100063). We acknowledge also financial support from the Spanish MEC (Grant No. FIS2011-65702-C02-01), 
ACI-Promociona (Grant No. ACI2009-1036), Grupos Consolidados UPV/EHU del Gobierno Vasco (Grant No. IT-319-07), and the European Research Council Advanced Grant DYNamo
(ERC-2010-AdG Proposal No. 267374). Finally, we thank Garnet K.-L. Chan, Simone Fratini, and Markus Holzmann for the help provided. *lorenzo.stella@ehu.es

${ }^{1}$ J. Hubbard, Proc. R. Soc. London, Ser. A 277, 237 (1964);

F. Gebhard, The Mott Metal-Insulator Transition: Models and Methods (Springer, Berlin, 1997).

${ }^{2}$ E. H. Lieb and F. Y. Wu, Phys. Rev. Lett. 20, 1445 (1968); Physica A 321, 1 (2003).

${ }^{3}$ J. Hachmann, W. Cardoen, and G. K.-L. Chan, J. Chem. Phys. 125, 144101 (2006).

${ }^{4}$ T. Tsuchimochi and G. E. Scuseria, J. Chem. Phys. 131, 121102 (2009).

${ }^{5}$ N. Lin, C. A. Marianetti, A. J. Millis, and D. R. Reichman, Phys. Rev. Lett. 106, 096402 (2011).

${ }^{6}$ A. V. Sinitskiy, L. Greenman, and D. A. Mazziotti, J. Chem. Phys. 133, 014104 (2010); D. A. Mazziotti, Phys. Rev. Lett. 106, 083001 (2011).

${ }^{7}$ R. Resta and S. Sorella, Phys. Rev. Lett. 82, 370 (1999).

${ }^{8}$ M. Capello, F. Becca, M. Fabrizio, S. Sorella, and E. Tosatti, Phys. Rev. Lett. 94, 026406 (2005).

${ }^{9} \mathrm{We}$ compute the electronic ground state of the full nonrelativistic Hamiltonian with fixed atomic geometry, but without using pseudopotentials.

${ }^{10}$ A. Karpfen, Chem. Phys. Lett. 61, 363 (1979); M. Kertész, J. Koller, and A. Ažman, Phys. Rev. B 19, 2034 (1979)

${ }^{11}$ I. Affleck, J. Phys.: Condens. Matter 1, 3047 (1989).

${ }^{12}$ G. K.-L. Chan and S. Sharma, Annu. Rev. Phys. Chem. 62, 465 (2011).

${ }^{13}$ R. Cimiraglia and R. Resta, Int. J. Quantum Chem. 19, 301 (1981); V. Vetere, A. Monari, G. L. Bendazzoli, S. Evangelisti, and B. Paulus, J. Chem. Phys. 128, 024701 (2008).

${ }^{14}$ W. M. C. Foulkes, L. Mitas, R. J. Needs, and G. Rajagopal, Rev. Mod. Phys. 73, 33 (2001).

${ }^{15}$ M. Casula, C. Filippi, and S. Sorella, Phys. Rev. Lett. 95, 100201 (2005); M. Casula, S. Sorella, and G. Senatore, Phys. Rev. B 74, 245427 (2006).

${ }^{16}$ P. Umari, A. J. Willamson, G. Galli, and N. Marzari, Phys. Rev. Lett. 95, 207602 (2005); P. Umari and N. Marzari, J. Chem. Phys. 131, 094101 (2009).
${ }^{17}$ N. D. Drummond, M. D. Towler, and R. J. Needs, Phys. Rev. B 70, 235119 (2004); P. Seth, P. López Ríos, and R. J. Needs, J. Chem. Phys. 134, 084105 (2011).

${ }^{18}$ M. Casula, C. Attaccalite, and S. Sorella, J. Chem. Phys. 121, 7110 (2004).

${ }^{19}$ M. Marchi, S. Azadi, M. Casula, and S. S. Sorella, J. Chem. Phys. 131, 154116 (2009); S. Azadi, C. Cavazzoni, and S. Sorella, Phys. Rev. B 82, 125112 (2010).

${ }^{20}$ S. Sorella, M. Casula, and D. Rocca, J. Chem. Phys. 127, 014105 (2007).

${ }^{21}$ S. Sorella, M. Casula, L. Spanu, and A. Dal Corso, Phys. Rev. B 83, 075119 (2011).

${ }^{22}$ C. J. Umrigar, J. Toulouse, C. Filippi, S. Sorella, and R. G. Hennig, Phys. Rev. Lett. 98, 110201 (2007).

${ }^{23}$ F. R. Petruzielo, J. Toulouse, and C. J. Umrigar, J. Chem. Phys. 132, 094109 (2010).

${ }^{24}$ Since expectation values of the variational wave function are not computed analytically, but stochasticly (Ref. 14), both Gaussiantype orbitals or Slater-type orbitals can be used with the same efficiency.

${ }^{25} \mathrm{~S}$. Sorella et al., [http://qe-forge.org/projects/turborvb/].

${ }^{26}$ W. Kohn, Phys. Rev. A 133, 171 (1964); R. Resta, Rev. Mod. Phys. 66, 899 (1994). N. D. Hine and W. M. C. Foulkes, J. Phys.: Condens. Matter 19, 506212 (2007).

${ }^{27}$ The original results from Table VI of Ref. 3 have been smoothly interpolated and uniformly shifted to obtain the reference of Fig. 1(a).

${ }^{28}$ H. J. Schulz, Phys. Rev. Lett. 64, 2831 (1990); M. Fabrizio, Phys. Rev. B 54, 10054 (1996); M. Capello, F. Becca, S. Yunoki, M. Fabrizio, and S. Sorella, ibid. 72, 085121 (2005).

${ }^{29}$ M. Calandra Buonaura and S. Sorella, Phys. Rev. B 57, 11446 (1998).

${ }^{30}$ T. Miyagawa and H. Yokoyama, Physica C 471, 738 (2011).

${ }^{31}$ N. F. Mott, Proc. Phys. Soc., London, Sect. A 62, 416 (1949).

${ }^{32}$ H.-H. Lai and O. I. Motrunich, Phys. Rev. B 81, 045105 (2010).

${ }^{33}$ H. J. Schulz, Phys. Rev. Lett. 71, 1864 (1993); A. R. Goñi, A. Pinczuk, J. S. Weiner, J. M. Calleja, B. S. Dennis, L. N. Pfeiffer, and K. W. West, ibid. 67, 3298 (1991). 